\title{
Hybrid Total Variation and Wavelet Thresholding Speckle Reduction for Medical Ultrasound Imaging
}

\author{
Banazier A. Abrahim*, Zeinab A. Mustafa, Inas A. Yassine, \\ Nourhan Zayed, and Yasser M. Kadah \\ Biomedical Engineering Department, Cairo University, Giza 12613, Egypt
}

\begin{abstract}
Ultrasound imaging is a widely used and safe medical diagnostic technique, due to its noninvasive nature, low cost, capability of forming real time imaging, and the continuing improvements in image quality. However, the usefulness of ultrasound imaging is degraded by the presence of signal dependent noise known as speckle. In this paper, we propose a new method for speckle reduction and coherence enhancement of ultrasound images based on a hybrid of total variation (TV) method and wavelet thresholding. In this model, a noisy image is decomposed into four subbands in wavelet domain. The low frequency subband contains the low frequency coefficients with less noise that can be easily eliminated using TV-based method. More edges and other detailed information like textures are contained in the other three subbands the wavelet based soft thresholding is applied on these three subbands. In the last step we use TV method to get the final denoised image since the TV is the ability of preserving edge is smoothening by wavelet thresholding. The proposed method is compared with previous methods as applied to simulated and real data using quantitative quality evaluation metrics to show the advantage of the new method.
\end{abstract}

Keywords: Ultrasound Imaging, Speckle Reduction, Total Variation, Wavelet Thresholding.

\section{INTRODUCTION}

Ultrasound imaging is a widely used and safe medical diagnostic technique and is often preferred over other medical imaging modalities, due to its noninvasive nature, low cost, capability of forming real time imaging, and the continuing improvements in image quality. ${ }^{1}$ However, the main weakness of medical ultrasound image is the poor quality of images, mainly due to multiplicative speckle noise that degrades the visual evaluation in ultrasound imaging. ${ }^{2}$

The presence of speckle noise in ultrasound images has been studied and documented since the early 1970s when researchers such as Burckhardt, ${ }^{3}$ Wagner et al. ${ }^{4}$ and Goodman ${ }^{5}$ described the fundamentals and the statistical properties of the speckle noise. Speckle is not really noise in the typical engineering sense because its texture often carries useful information about the image being viewed. It is the primary factor that limits the contrast resolution in diagnostic ultrasound imaging, thereby limiting the detectability of small, low-contrast lesions and making the ultrasound images generally difficult for the non specialist to interpret. ${ }^{3,4,6,7}$ Speckle noise also limits the effective application of image processing and analysis algorithms (i.e., edge detection, segmentation) and display in two-dimensional (2-D) and volume rendering in 3-D. Therefore, speckle is most often considered a dominant source of noise in ultrasound imaging and needed to be

${ }^{*}$ Author to whom correspondence should be addressed. filtered out ${ }^{3,6,7}$ without affecting important features and texture of the image.

Speckle in ultrasound $B$-scans is seen as a granular structure which is caused by the constructive and destructive coherent interferences of back scattered echoes from the scatterers that are typically much smaller than the spatial resolution of medical ultrasound system. This phenomenon is common to laser, sonar and synthetic aperture radar imagery (SAR). Speckle pattern is a form of multiplicative noise and that depends on the structure of imaged tissue and various imaging parameters. Speckle degrades the target delectability in $B$-scan images and reduces the contrast, resolutions which affect the human ability to identify normal and pathological tissue. It also degrades the speed and accuracy of ultrasound image processing tasks such as segmentation and registration. There are two main purposes for speckle reduction in medical ultrasound imaging: (i) improve the human interpretation of ultrasound images, (ii) preprocessing step for many ultrasound image processing tasks such as segmentation and registration. ${ }^{8}$

In literature many techniques have been studied speckle noise reduction. The medain filter (median) is a simple nonlinear operator that replaces the middle pixel in the window with the medianvalue of its neighbors. ${ }^{9}$ Diffusion filters ( $\mathrm{srad}$ ) remove noise from an image by modifying the image via solving a partial differential equation (PDE). The smoothing of srad is carried out, depending on the image edges and their directions. Anisotropic diffusion is an efficient, nonlinear technique for simultaneously performing contrast enhancement and noise reduction. ${ }^{10}$ The concept of 
the geometric filtering is that speckle appears in the image as narrow walls and valleys. The Geometric despeckle filter $(g f 4 d)$, through iterative repetition, that gradually tears down the narrow walls (bright edges) and fills up the narrow valleys (dark edges), thus smearing the weak edges that need to be preserved. ${ }^{11}$ First Order Statistics Filtering, (lsmv) Mean and variance local statistics despeckle filter this filter uses the first order statistics such as the variance and the mean of the neighborhood. ${ }^{12}$

The image denoising is a field where one is frequently interested in removing noise without sacrificing important structures such as edges. Since this is not possible with linear techniques many nonlinear strategies have been proposed in the last two decades, also image denoising problem has been widely studied and two main approaches have been developed: the variational methods ${ }^{13-17}$ and wavelet techniques. ${ }^{18-24}$ Among variational approaches, a classical variational denoising algorithm is the total variation minimization problem of Rudin-Osher-Fatemi $(\mathrm{ROF}):^{14}$

$$
\operatorname{MIN}_{u}\left\{J(u)+(2 \lambda)^{-1}\|f-u\|_{2}^{2}\right\}
$$

where $f$ is the noisy image, $u$ is the image we want to restore from $f$. The constant $\lambda>0$ is a turning parameter; larger value of $\lambda$ gives blurring effect to the image. $J(u)=\int|\nabla u|$ is often referred to as total variation (TV). So, this method tries to remove local variations while maintaining the 2-norm distance to the original image as small as possible to preserve major features. The major advantage of the classical TV model is the ability of preserving edges and fine structures while removing the noise and artifacts. However, these TV-based methods undergo several iterations for denoising. Thus, estimating the number of iterations is the main disadvantage in TV-based denoising. ${ }^{25}$

The wavelet techniques are widely used in the image processing, such as the image compression, image de-noising. It has been shown that its performance of image processing is better than the methods based on other linear transformation. The wavelet de-noising method decomposes the image into the wavelet basis and shrinks the wavelet coefficients in order to despeckle the image. Wavelet soft-thresholding method is another popular denoising method in image processing introduced by Donoho et al. was studied and extended in several papers. ${ }^{19-21}$ They have introduced a universal threshold $T$, given by:

$$
T=\sqrt{2 \sigma^{2} \log N}
$$

where, $\sigma^{2}$ is the noise variance and $N$ is the total number of pixels. The use of this universal threshold to denoise images in wavelet domain is known as VisuShrink. ${ }^{20}$

In wavelet thresholding, the image is decomposed into approximation (low-frequency) and detail (high-frequency) subbands, and the coefficients in the detail subbands are processed via hard or soft thresholding. The hard thresholding eliminates (sets to zero) coefficients that are smaller than a threshold; the soft thresholding shrinks the coefficients that are larger than the threshold as well. The main task of the wavelet thresholding is the selection of threshold value and the effect of denoising depends on the selected threshold: a bigger threshold will throw off the useful information and the noise components at the same time while a smaller threshold cannot eliminate the noise effectively. ${ }^{26}$

There has also been a rapidly increasing interest in designing hybrid methods that uses both wavelet and TV denoising methods. ${ }^{27-31}$ Durand et al. ${ }^{28}$ proposed a hybrid method to remove the pseudo-Gibbs phenomenon by replacing the thresholded coefficients by values minimizing the TV. Based on a similar idea, Chan et al. ${ }^{29}$ postprocessed images obtained from wavelet shrinkage by a TV regularization technique. Coifman et al $^{30}$ postprocessed the signals that have been degraded by wavelet thresholding by solving functional minimization problems with wavelet constraints.

The aim of the hybrid method proposed in this paper is to resolve the contradiction between speckle noise suppression and texture and edges image preserving, which cannot be resolved by the TV-based method or wavelet method independently. The TV model is the best, it is able to sufficiently remove spatially adaptive noise and artifacts while preserving edges and fine structures, also a major strength of the wavelet thresholding is the ability to treat different frequency components of an image separately; this is important, because noise in real scenarios may be frequency dependent. But, in wavelet thresholding the problem experienced is generally smoothening of edges. Which mean that the wavelet approach and TV-based method were combined not only to remove the noise, but also preserve the details, edges and textures.

The paper is organized as follows. Section 2, explains material and method description which consists of total variation, wavelet decomposition, Wavelet thresholding, hybrid denoising method, algorithm and image quality evaluation metrics. Section 3 depicts the qualitative and quantitative comparison among different filters showing the experimental results. Section 4 presents a discussion of the method. Finally, the conclusion is presented in Section 5.

\section{MATERIALS AND METHOD DESCRIPTION}

\subsection{Total Variation}

Because of its virtue of preserving sharp edges, it is widely used in many applications of image processing. Rudin et al. ${ }^{14}$ solved the total variation minimization problem through PDE-based schemes, which is numerical intensive. Besides, Chambolle's projection algorithm ${ }^{15}$ is a fast method to solve the ROF model given as,

$$
u=f-P_{G_{\lambda}}(f)
$$

where $f$ is the noisy image, $u$ is the image we want to restore from $f, G_{\lambda}=\left\{v \in G /\|v\|_{G} \leq \lambda\right\}, P_{G_{\lambda}}(f)$ is the orthogonal projection of $f$ on $G_{\lambda}$ and the space $G$ is proposed by Meyer for modeling oscillating patterns. ${ }^{13}$

\subsection{Wavelet Decomposition}

The wavelet decomposition process involves three basic steps as follows:

(1) Linear forward wavelet transform

(2) Nonlinear thresholding step conversion of wavelets coefficients with threshold/shrinkage function

(3) Linear inverse wavelet transform.

\subsection{Wavelet Thresholding}

The wavelet-tresholding denoising methods modify each wavelet coefficient by threshold function.

Let $f=\left\{f_{i j}, i, j=1,2 \ldots M\right\}$ denote the $M \times M$ matrix of the original image and $M$ is some integer power of 2. During transmission the image $f$ is corrupted by white Gaussian noise with independent and identically distributed (i.i.d.) zero mean, and standard deviation $\sigma$ i.e., $n_{i j} \sim N\left(0, \sigma^{2}\right)$. So, the noisy 


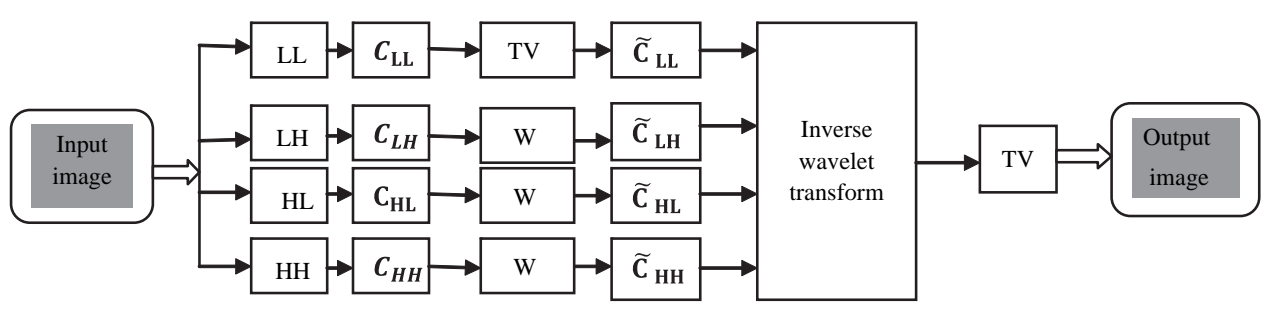

Fig. 1. Block diagram of the proposed despecking method. First, the input noisy image decompose into four subbands of $\mathrm{LL}$, $\mathrm{LH}, \mathrm{HL}, \mathrm{HH}$ get the corresponding wavelet coefficients $C_{\mathrm{LL}}, C_{\mathrm{LH}}, C_{\mathrm{HL}}, C_{\mathrm{HH}}$. Then apply total variation method (TV) to the $\mathrm{LL}$ subband $\left(C_{\mathrm{LL}}\right)$ to get $C_{\mathrm{LL}}$ also apply the wavelet thresholding $(\boldsymbol{W})$ to $C_{\mathrm{LH}}, C_{\mathrm{HL}}, C_{\mathrm{HH}}$, and get the modified wavelet $\tilde{C}_{\mathrm{LH}}, \tilde{C}_{\mathrm{HL}}, \tilde{C}_{\mathrm{HH}}$. After that, apply inverse wavelet transform on $\tilde{C}_{\mathrm{LL}}, \tilde{C}_{\mathrm{LH}}, \tilde{C}_{\mathrm{HL}}, \tilde{C}_{\mathrm{HH}}$ and get the reconstructed image. Finally, apply TV to reconstructed image to get the final denoised image.

image received at the receiver end is $g_{i j}=f_{i j}+\sigma n_{i j}$. The goal is to estimate the signal $f$ from noisy observations $g_{i j}$. Let $W$ and $W^{-1}$ denote the two dimensional orthogonal discrete wavelet transform (DWT) matrix and its inverse respectively. Then, $Y=$ $W \cdot g$ represents the matrix of wavelet coefficients of $g$ having four subbands (LL, LH, HL and HH). The sub-bands $H H_{k}, H L_{k}$, and $L H_{k}$ are called details, where $K$ is the scale varying from $1,2 \ldots J$ and $J$ is the total number of decompositions. The size of the subband at scale $K$ is $N / 2 K \times N / 2 K$. The subband $L L_{J}$ is the low-resolution residue. The wavelet thresholding denoising method processes each coefficient of $Y$ from the detail subbands with a soft threshold function to obtain $\hat{X}^{26}$ The denoised estimate is inverse transformed to

$$
\hat{f}=W^{-1} \hat{X}
$$

The basic Procedure for all thresholding method is as follows:

(i) Calculate the DWT of the image.

(ii) Threshold the wavelet coefficients.

(iii) Compute the IDWT to get the denoised estimate.

There are two thresholding functions frequently used, i.e., a hard threshold, a soft threshold.

The hard-thresholding is described as

$$
\eta_{1}(w)=w I(|w|>T)
$$

Where $w$ is a wavelet coefficient, $T$ is the threshold.

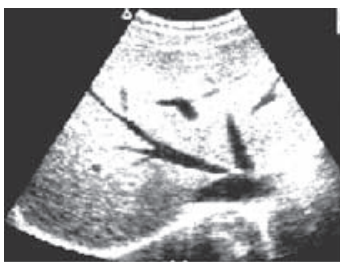

(a) LL suband

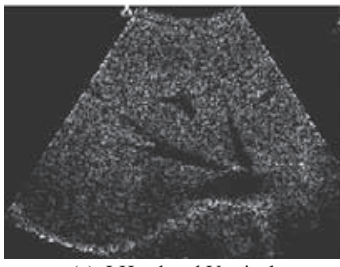

(c) LH suband Vertical

Direction

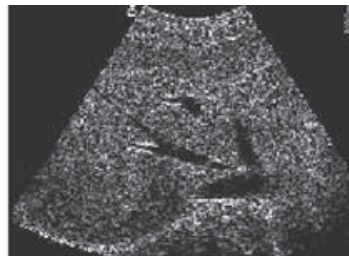
Direction

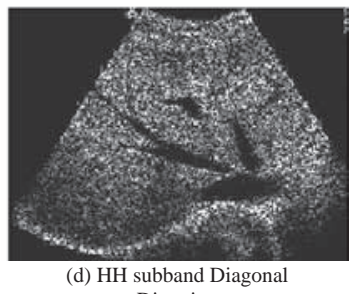

Direction (b) HLsuband Horizontal

Fig. 2. Four subbands obtained by wavelet decomposition (liver image).
The soft-thresholding function is described as

$$
\eta_{2}(w)=(w-\operatorname{sgn}(w) T) I(|w|>T)
$$

Here $\operatorname{sgn}(x)$ is the sign function of $x .^{32}$

In the algorithm we are proposing, soft thresholding has been used over hard thresholding because it gives more visually pleasant images as compared to hard thresholding; since the latter is discontinuous and yields abrupt artifacts in the recovered images especially when the noise energy is significant. The value of softthresholding in the proposed algorithm is set at the same value obtained with wavelet thresholding only, ${ }^{26}$ the best result in this model was obtained when the threshold value is 0.01 using "db8" filters.

\subsection{Hybrid Denoising Method}

Let $f$ be an input image corrupted by the speckle noise. The wavelet representation of $f$ can be described in terms of scaling and wavelet functions as:

$$
f=\sum_{k \in Z^{2}}\left\langle f, \varphi_{0, k}\right\rangle \varphi_{0, k}+\sum_{k \in Z^{2}} \sum_{j=0}^{\infty} \sum_{i=1}^{3}\left\langle f, \psi_{j, k}^{i}\right\rangle \psi_{j, k}^{i}
$$

where $\varphi$ is the scaling function, and $\psi$ are a wavelet function, $\psi=\left\{\psi^{1}, \psi^{2}, \psi^{3}\right\}$ are two-dimensional wavelets constructed by

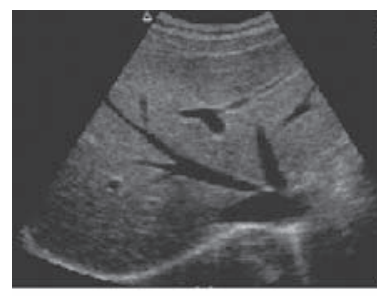

(a) LL subband after denoising (TV)

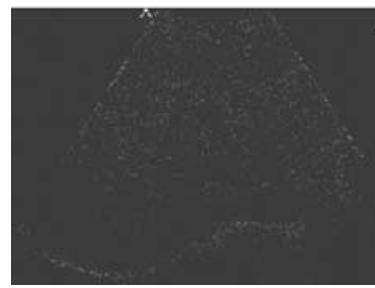

(c) LH subband after thresholding method

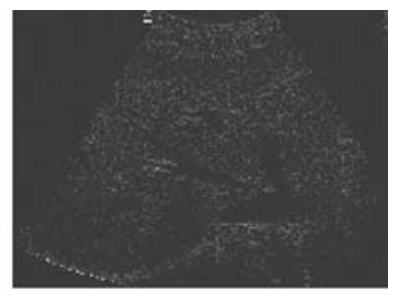

(b) HL subband after thresholding method

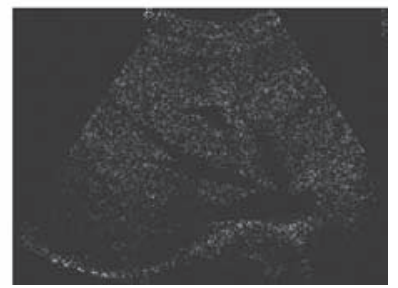

(d) HH subband after thresholding method
Fig. 3. Modified subbands obtained by apply TV to LL subband and wavelet thresholding to another subbands (liver image). 


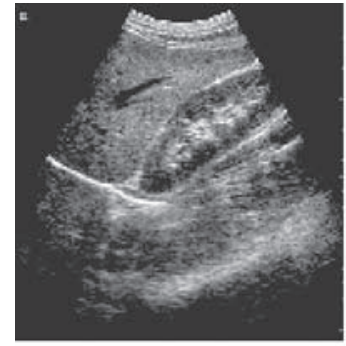

(a)

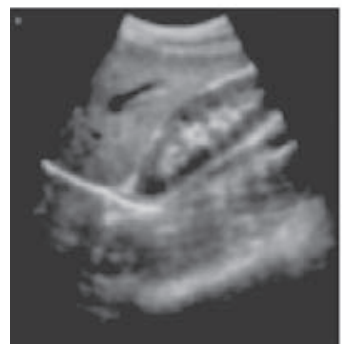

(e)

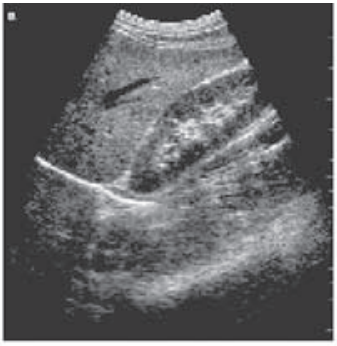

(b)

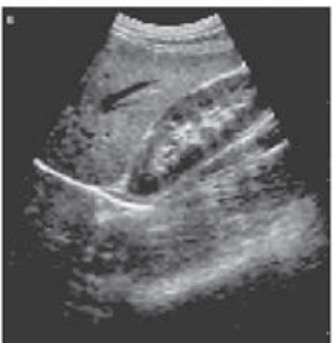

(f)

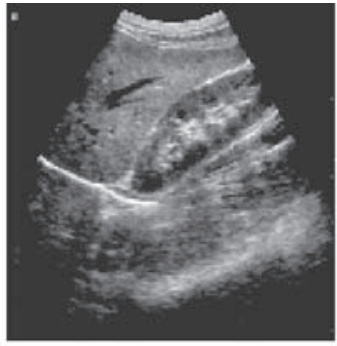

(c)

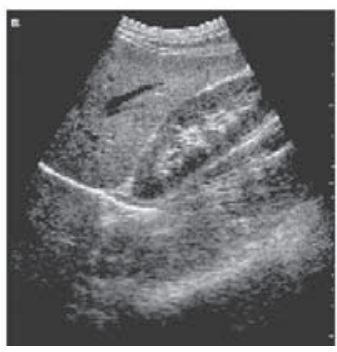

(g)

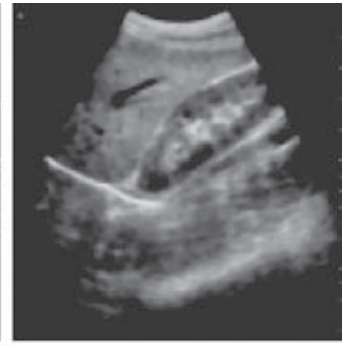

(d)

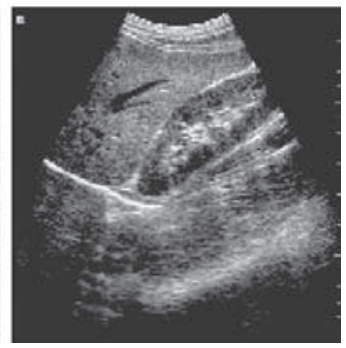

(h)

Fig. 4. Qualitative results of various filters; original abdominal ultrasound image given in (a), median filter in (b), speckle reducing anisotropic diffusion filtering $(s r a d)$ in (c), geometric despeckle filter $(g f 4 d)$ in (d), mean and variance local statistics despeckle filter (Ismv) in (e), wavelet Thresholding in (f), total variation despeckle filter in $(\mathrm{g})$, and proposed method in $(\mathrm{h})$.

tensor products of a one-dimensional orthogonal wavelet system, where $\psi^{1}$ is the vertical, $\psi^{2}$ the horizontal and $\psi^{3}$ is the diagonal. We decompose the image to one level, which results in LL, HL, LH and HH subbands, i.e.,

$$
f=\sum_{k \in Z^{2}}\left\langle f, \varnothing_{0, K}\right\rangle \varnothing_{0, K}+\sum_{k \in Z^{2}} \sum_{i=1}^{3}\left\langle f, \psi_{j, k}^{i}\right\rangle \psi_{0, K}^{i}
$$

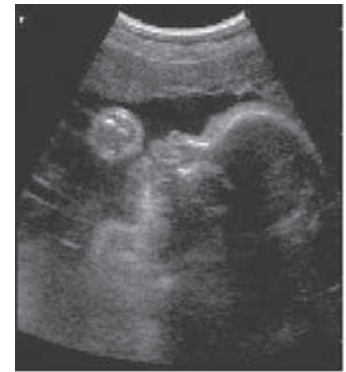

(a)

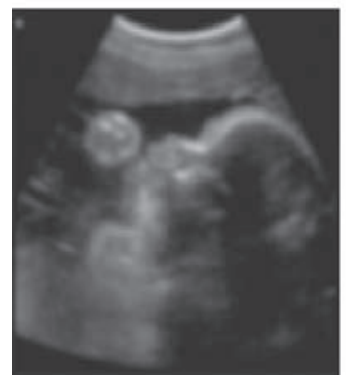

(e)

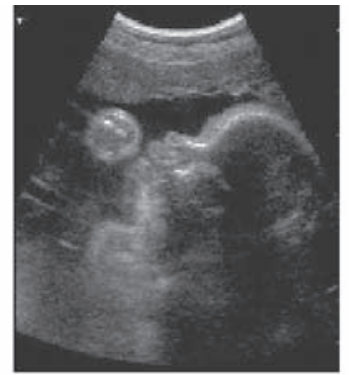

(b)

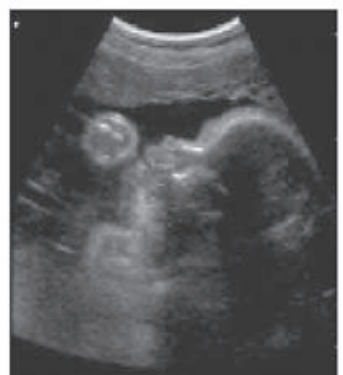

(f)
We denote the wavelet coefficients corresponding to the four subbands by:

$$
\begin{aligned}
C_{\mathrm{LL}}=\left\langle f, \varphi_{0, k}\right\rangle ; & C_{\mathrm{LH}}=\left\langle f, \psi_{0, K}^{1}\right\rangle \\
C_{\mathrm{HL}}=\left\langle f, \psi_{0, k}^{2}\right\rangle ; & C_{\mathrm{HH}}=\left\langle f, \psi_{0, K}^{3}\right\rangle
\end{aligned}
$$

where $C_{\mathrm{LL}}$ is called low frequency subband, $C_{\mathrm{LH}}, C_{\mathrm{HL}}, C_{\mathrm{HH}}$ are called high frequency subbands of vertical, horizontal, and

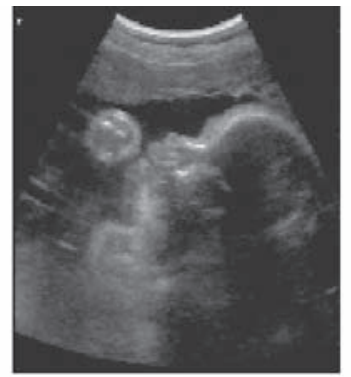

(c)

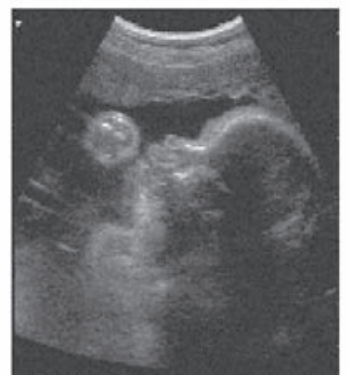

(g)

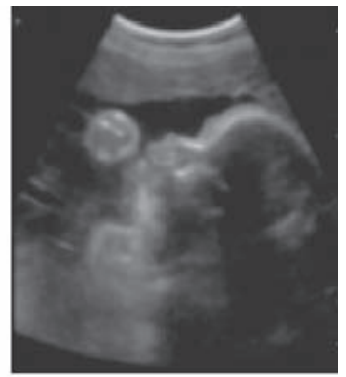

(d)

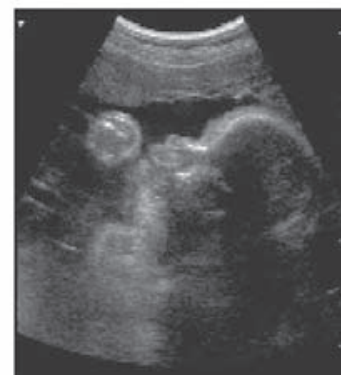

(h)

Fig. 5. Qualitative results of various filters; original obstetrical ultrasound image given in (a), median filter in (b), speckle reducing anisotropic diffusion filtering $(s r a d)$ in (c), geometric despeckle filter $(g f 4 d)$ in (d), mean and variance local statistics despeckle filter (Ismv) in (e), wavelet thresholding in (f), total variation despeckle filter in $(\mathrm{g})$, and proposed method in $(\mathrm{h})$. 


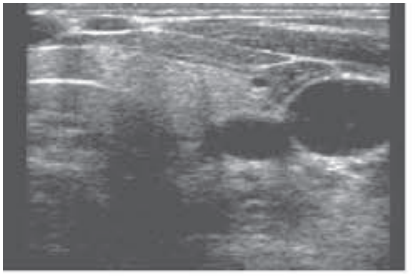

(a)

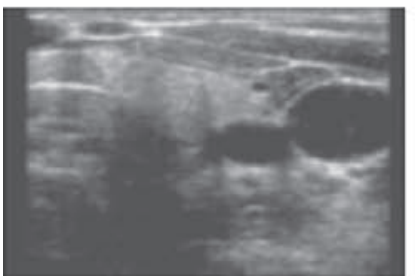

(e)

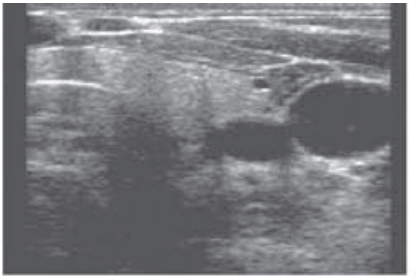

(b)

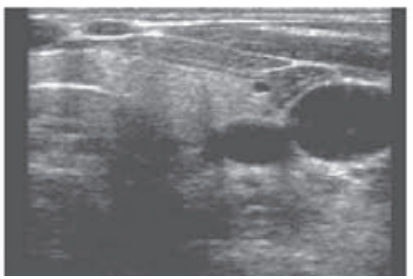

(f)

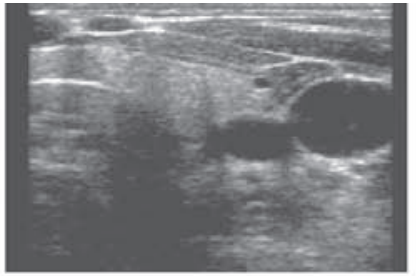

(c)

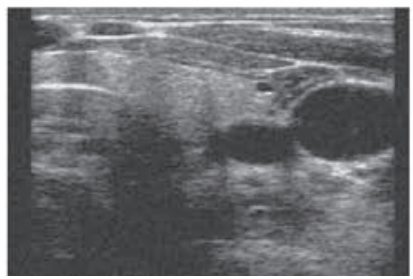

(g)

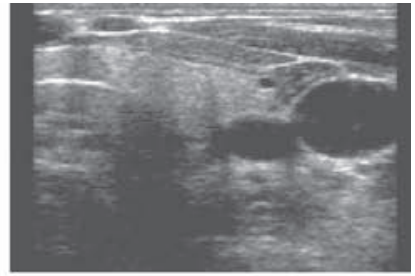

(d)

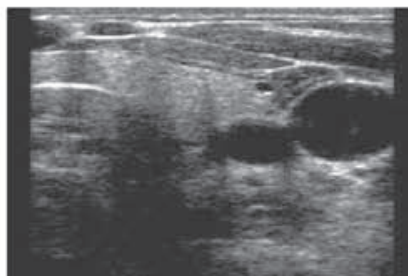

(h)

Fig. 6. Qualitative results of various filters; original small parts ultrasound image given in (a), median filter in (b), speckle reducing anisotropic diffusion filtering $(s r a d)$ in (c), geometric despeckle filter ( $g f 4 d)$ in (d), mean and variance local statistics despeckle filter (Ismv) in (e), wavelet thresholding in (f), total variation despeckle filter in $(\mathrm{g})$, and proposed method in (h).

diagonal directions, respectively. Since the wavelet coefficients of the noise and textures mainly concentrate on the high frequency subbands, we apply the soft wavelet thresholding to get modified wavelet coefficients $\tilde{C}_{\mathrm{LH}}, \tilde{C}_{\mathrm{HL}}, \tilde{C}_{\mathrm{HH}}$, the $\tilde{C}_{\mathrm{LL}}$ subband contains less noise which can be easily eliminated using total variation, so we can apply Chambolle's method to denoise the LL subband. Then we apply Chambolle's projection algorithm ${ }^{15}$ to LL subband and get the modified coefficient given by:

$$
\tilde{C}_{\mathrm{LL}}=C_{\mathrm{LL}}-P_{G_{\lambda}}\left(C_{\mathrm{LL}}\right)
$$

After obtaining the modified coefficients $\tilde{C}_{\mathrm{LL}}, \tilde{C}_{\mathrm{LH}}, \tilde{C}_{\mathrm{HL}}, \tilde{C}_{\mathrm{HH}}$ we apply inverse wavelet transform and get the reconstructed

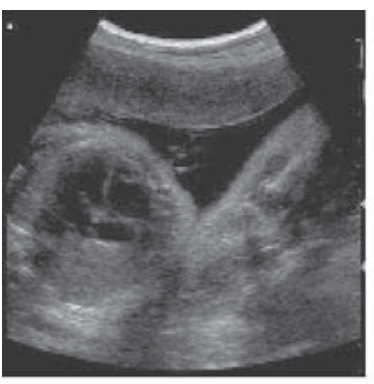

(a)

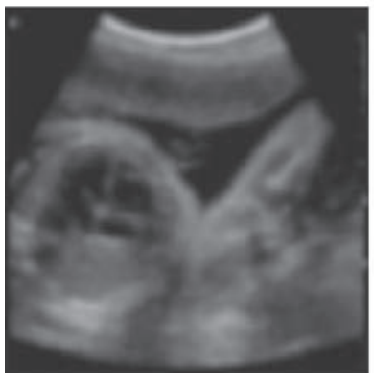

(e)

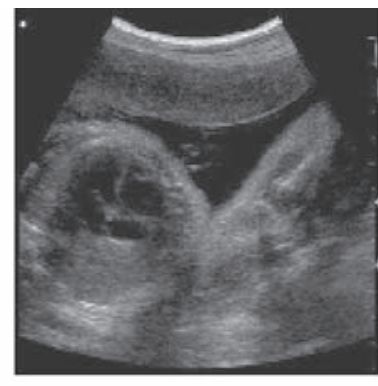

(b)

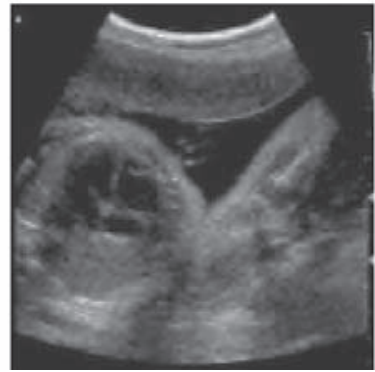

(f)

image, Finally TV method to reconstructed image is applied to get the final denoised image, Figure 1 summarized the proposed method in block diagram.

\subsection{Algorithm}

According to the analysis of the combined method given above, the proposed algorithm can be summarized as following:

Step 1: Decompose the input image into four subbands of LL, $\mathrm{LH}, \mathrm{HL}, \mathrm{HH}$ and get the corresponding wavelet coefficients $C_{\mathrm{LL}}$, $C_{\mathrm{LH}}, C_{\mathrm{HL}}, C_{\mathrm{HH}}$.

Step 2: Apply TV method to the LL subband and get $\tilde{C}_{\mathrm{LL}}$, i.e,

$$
\tilde{C}_{\mathrm{LL}}=C_{\mathrm{LL}}-P_{G_{\lambda}}\left(C_{\mathrm{LL}}\right)
$$

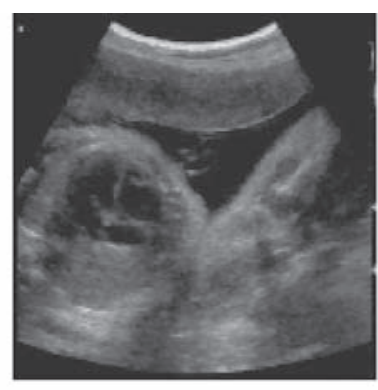

(c)

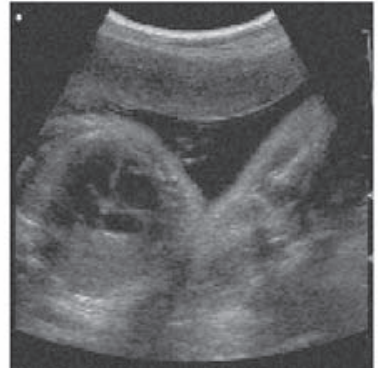

(g)

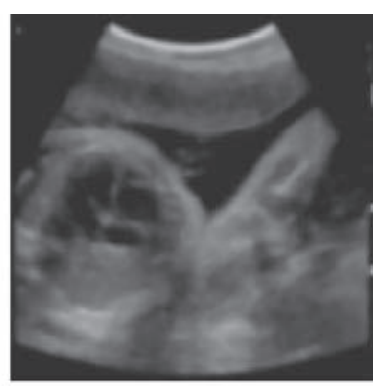

(d)

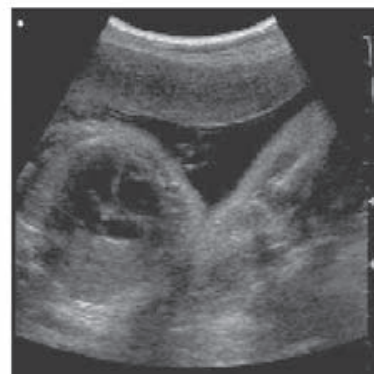

(h)

Fig. 7. Qualitative results of various filters; original abdominal ultrasound image given in (a), median filter in (b), speckle reducing anisotropic diffusion filtering $(s r a d)$ in (c), geometric despeckle filter ( $g f 4 d)$ in (d), mean and variance local statistics despeckle filter (Ismv) in (e), wavelet thresholding in (f), total variation despeckle filter in $(\mathrm{g})$, and proposed method in $(\mathrm{h})$. 


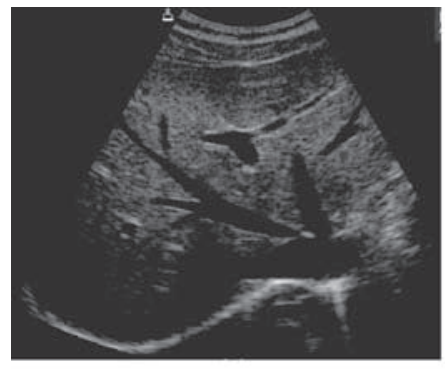

(a)

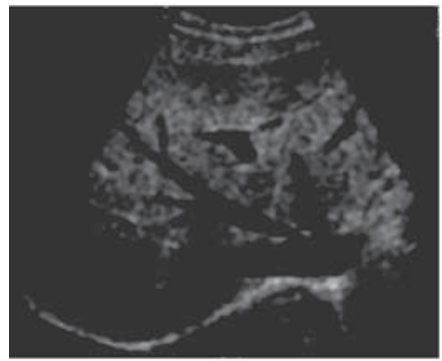

(d)

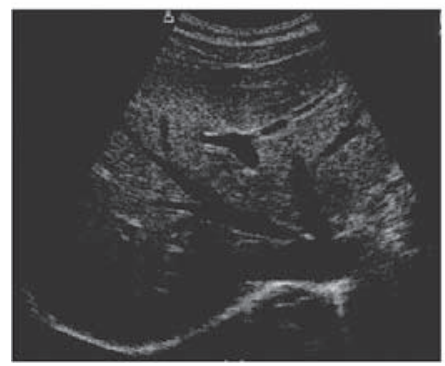

(g)

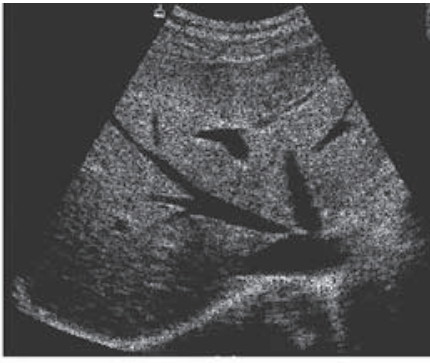

(b)

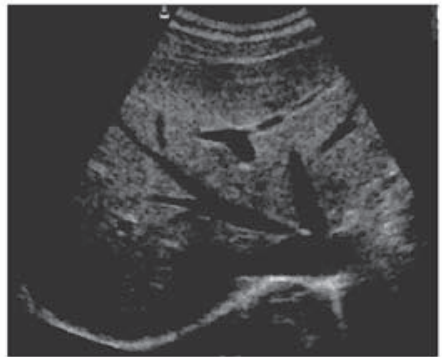

(e)

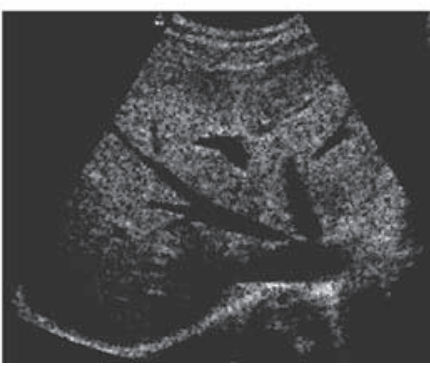

(h)

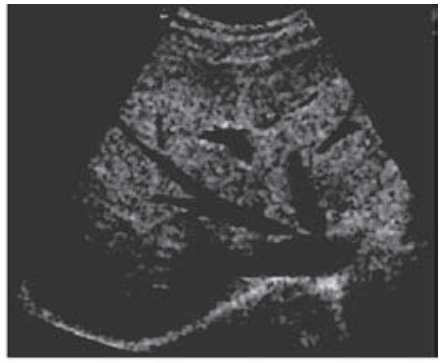

(c)

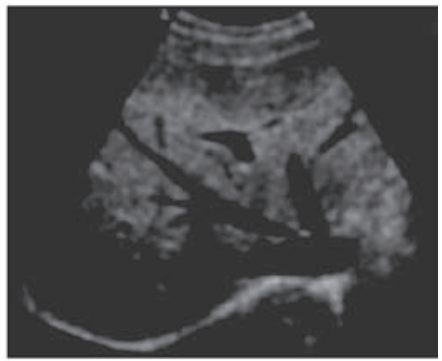

(f)

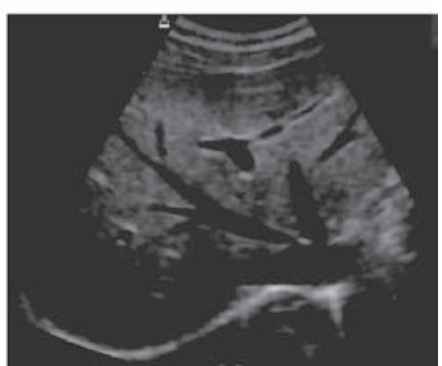

(i)

Fig. 8. Results of various filters on a multiplicative noise with $\sigma^{2}=0.05$ original ultrasound image (liver image) given in (a) noisy image given in (b), median filter (median) in (c), speckle reducing anisotropic diffusion filtering (srad) in (d), geometric despeckle filter ( $g f 4 d)$ in (e), mean and variance local statistics despeckle filter (Ismv) in (f), wavelet thresholding in $(\mathrm{g})$, total variation despeckle filter (h), and proposed method in (i).

Step 3: Apply the wavelet thresholding method to $C_{\mathrm{LH}}, C_{\mathrm{HL}}, C_{\mathrm{HH}}$ and compute the modified wavelet $\tilde{C}_{\mathrm{LH}}, \tilde{C}_{\mathrm{HL}}, \tilde{C}_{\mathrm{HH}}$.

Step 4: Apply inverse wavelet transform on $\tilde{C}_{\mathrm{LL}}, \tilde{C}_{\mathrm{LH}}, \tilde{C}_{\mathrm{HL}}$, $\tilde{C}_{\mathrm{HH}}$ and get the reconstructed image.

Step 5: Apply TV method to reconstructed image to get the final denoised image.

\subsection{Image Quality Evaluation Metrics}

To quantify the performance improvements of the speckle reduction method various measures may be used. The commonly preferred measures are mean squared error (MSE) and root mean squared error (RMSE), signal to noise ratio (SNR), peak signal to noise ratio (PSNR), Correlation coefficient (CoC), structural similarity index (SSIN), because the structural similarity index and Correlation coefficient are measure of similarity between the original and denoised images in this paper we used (SSIN), and universal quality index $Q .{ }^{8}$

In this paper the differences between the original, $g_{i, j}$, and the despeckled, $f_{i, j}$, images were evaluated using image quality evaluation metrics. The following measures, which are easy to compute and have clear physical meaning, were computed:
The MSE (Mean square error):

$$
\mathrm{MSE}=\frac{1}{M N} \sum_{i=1}^{M} \sum_{j=1}^{N}\left(g_{i, j}-f_{i, j}\right)^{2}
$$

which measures the quality change between the original and processed image in an $M \times N$ window. ${ }^{33}$

The signal-to-noise ratio (SNR) is given by: ${ }^{34}$

$$
\mathrm{SNR}=10 \log _{10} \frac{\sum_{i=1}^{M} \sum_{j=1}^{N}\left(g_{i, j}^{2}+f_{i, j}^{2}\right)}{\sum_{i=1}^{M} \sum_{j=1}^{N}\left(g_{i, j}-f_{i, j}\right)^{2}}
$$

The peak SNR (PSNR) is computed using: ${ }^{34}$

$$
\text { PSNR }=-10 \log _{10} \frac{\text { MSN }}{g_{\max }^{2}}
$$

where $g_{\max }^{2}$ is the maximum intensity in the unfiltered image. The PSNR is higher for a better-transformed image and lower for a poorly transformed image. It measures image fidelity, which is how closely the despeckled image resembles the original image.

The structural similarity index between two images, ${ }^{35}$ is given by:

$$
\operatorname{SSIN}=\frac{\left(2 \bar{g} f+c_{1}\right)\left(2 \sigma_{g f}+c_{2}\right)}{\left(\bar{g}^{2}+\bar{f}^{2}+c_{1}\right)\left(\sigma_{g}^{2}+\sigma_{f}^{2}+c_{2}\right)}, \quad-1<\operatorname{SSIN}<1
$$




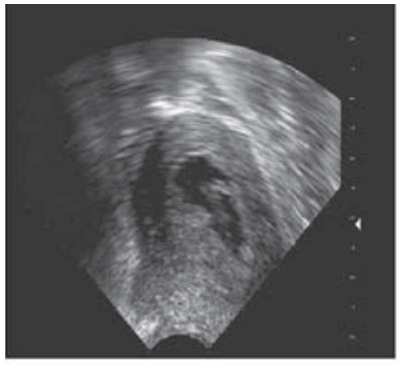

(a)

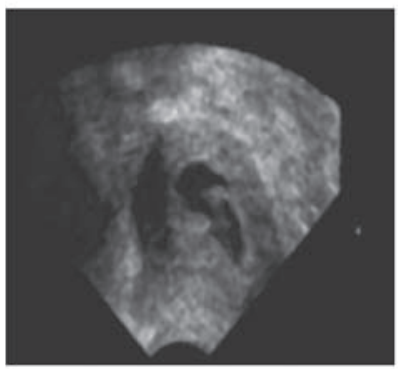

(d)

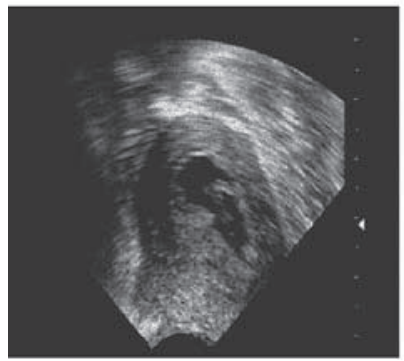

(g)

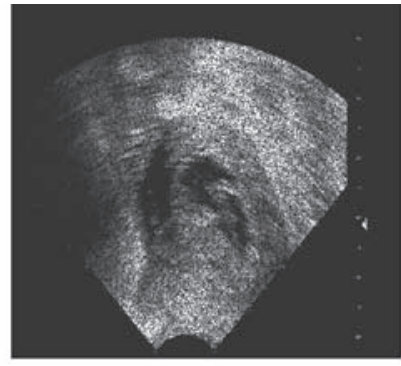

(b)

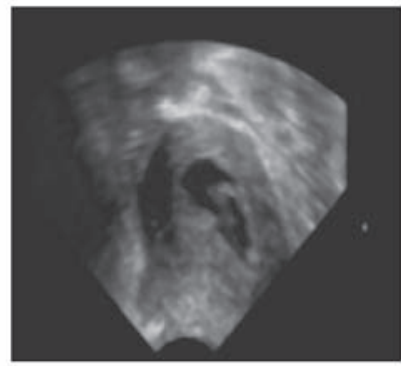

(e)

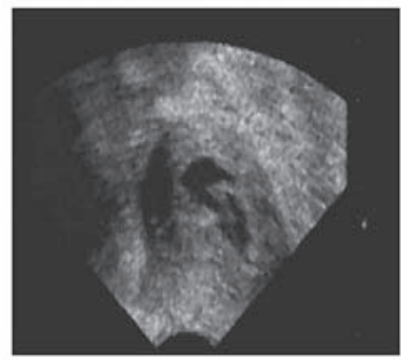

(h)

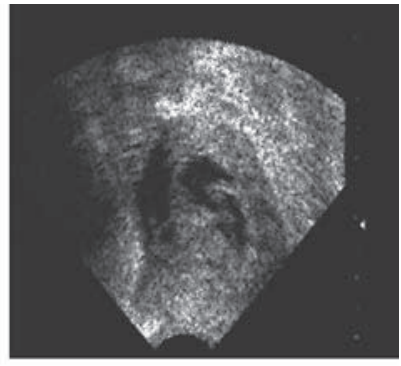

(c)

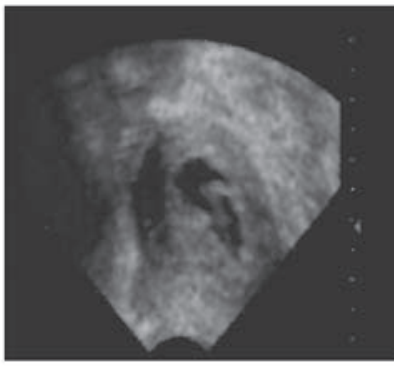

(f)

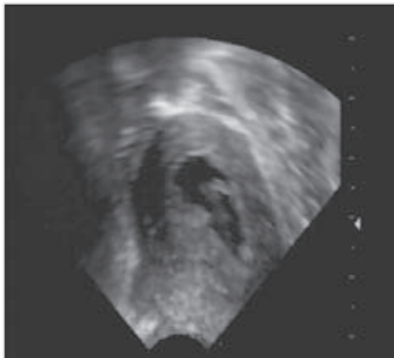

(i)

Fig. 9. Results of various filters on a multiplicative noise with $\sigma^{2}=0.05$ original ultrasound image (trans-vaginal image) given in (a) noisy image given in (b), median filter (median) in (c), speckle reducing anisotropic diffusion filtering (srad) in (d), geometric despeckle filter ( $g f 4 d$ ) in (e), mean and variance local statistics despeckle filter (Ismv) in (f), wavelet thresholding in (g), total variation despeckle filter (h), and proposed method in (i).

where $\bar{g}$ and $\bar{f}$ represent the mean of the original and despeckled values with their standard deviations, $\sigma_{g}$ and $\sigma_{f}$, of the original and despeckled values of the analysis window, and $\sigma_{g f}$ represents the covariance between the original and despeckled windows, $c_{1}=0.01 d r$ and $c_{2}=0.03 d r$, with $d r=255$ representing the dynamic range of the ultrasound images. The range of values for the SSIN lies between -1 , for bad and 1 for good similarity between the original and despeckled images, respectively.

The mathematically defined universal quality index ${ }^{36}$ models any distortion as a combination of three different factors: loss of correlation, luminance distortion, and contrast distortion and is derived as:

$$
Q=\frac{\sigma_{g f}}{\sigma_{f} \sigma_{g}}, \frac{2 f \bar{g}}{(\bar{f})^{2}+(\bar{g})^{2}}, \frac{2 \sigma_{f} \sigma_{g}}{\sigma_{f}^{2}+\sigma_{g}^{2}}, \quad-1<Q<1
$$

Its highest value is 1 if $g_{i, j}=f_{i, j}$; its lowest value is -1 if $f_{i, j}=2 \bar{g}-g_{i, j}$.

\section{EXPERIMENTAL RESULTS}

The proposed method is implemented and qualitative and quantitatve evaluations of the method were performed. In the qualitative performance evaluation study, a total of ten ultrasound images from the IBE Tech (Giza, Egypt) database of ultrasound images $^{38}$ of different resolutions comprising different applications including abdominal, obstetrical, small parts and endocavity imaging were processed and the results were evaluated by the IBE Tech experts. In the quantitative study, ten specklefree ultrasound images from different sources ${ }^{37-41}$ and for different applications were artificially corrupted by speckle noise with variance $\sigma^{2}=0.05$ using the MATLAB command "imnoise (image, 'speckle,' 0.05)." This value was used since it represents a practical value for speckle variance in ultrasound image taken in clinical settings. This allows the quantitative evaluation to have a gold standard or a reference image to compare the results to. A Monte Carlo simulation with a total of ten independent runs each with different speckle noise pattern was performed for each image. The performance of noise removing algorithms is measured using quantitative performance measures such as MSE, SNR, PSNR, SSIN, and $Q$ averaged over all runs for same image. It should be noted that speckle free images can be obtained using acquisition-based methods such as spatial or frequency compounding. Here, eight examples from these quantitative and qualitative studies are reported and the performance of the new method is compared with six other techniques; namely, median, speckle reducing anisotropic diffusion ( $\mathrm{srad}$ ), geometric 


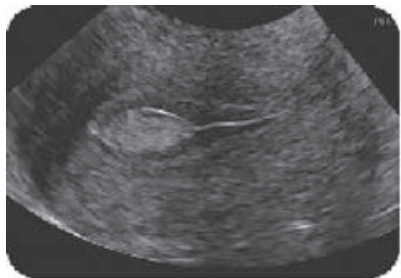

(a)

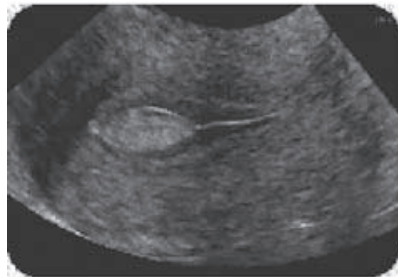

(d)

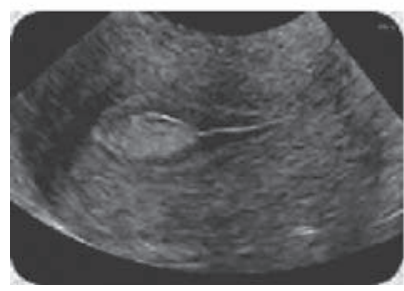

(g)

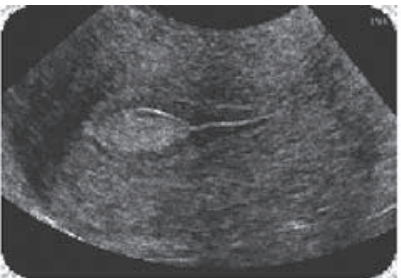

(b)

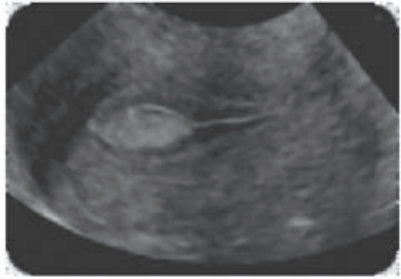

(e)

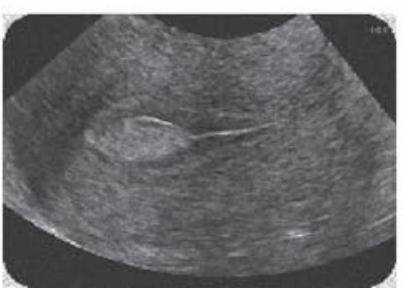

(h)

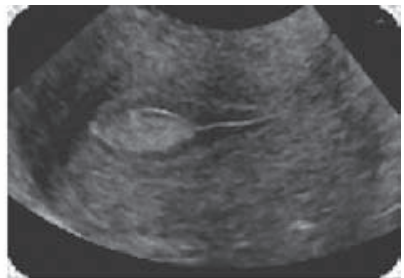

(c)

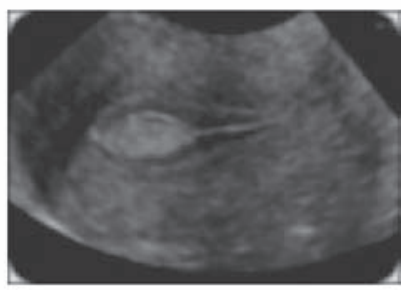

(f)

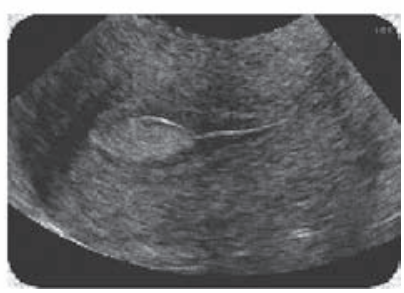

(i)

Fig. 10. Results of various filters on a multiplicative noise with $\sigma^{2}=0.05$ original ultrasound image (pelvic image) given in (a) noisy image given in (b), median filter (median) in (c), speckle reducing anisotropic diffusion filtering (srad) in (d), geometric despeckle filter ( $g f 4 d)$ in (e), mean and variance local statistics despeckle filter (Ismv) in (f), wavelet thresholding in $(\mathrm{g})$, total variation despeckle filter (h), and proposed method in (i).

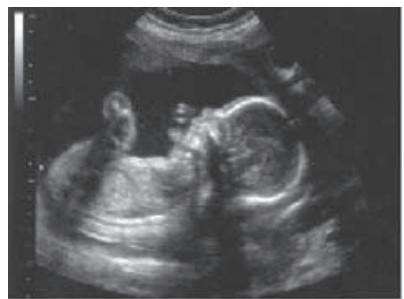

(a)

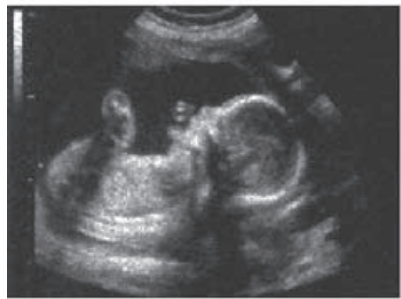

(d)

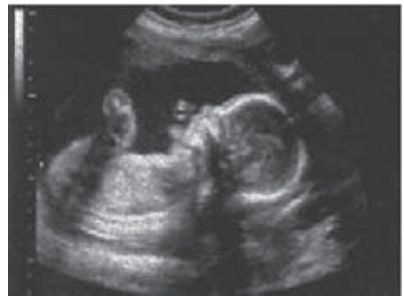

(g)

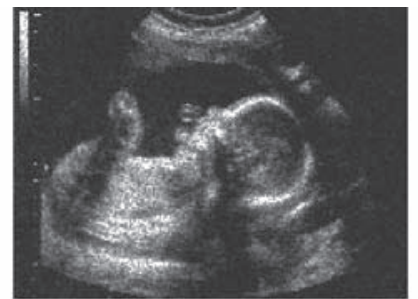

(b)

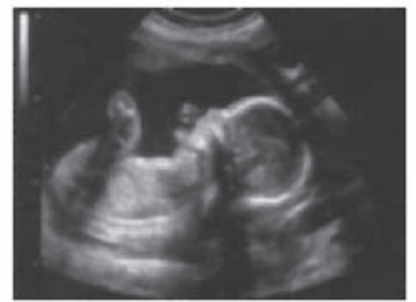

(e)

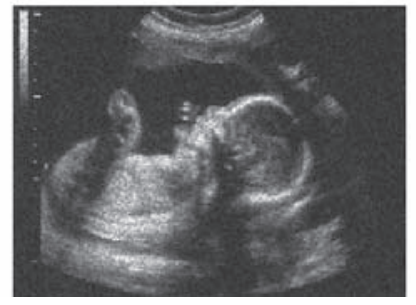

(h)

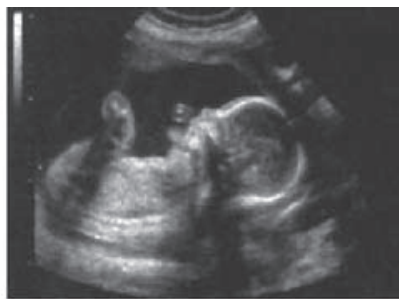

(c)

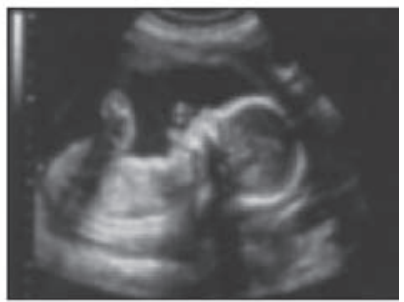

(f)

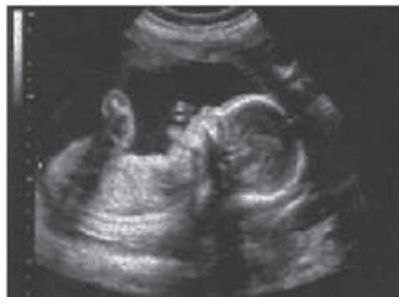

(i)

Fig. 11. Results of various filters on a multiplicative noise with $\sigma^{2}=0.05$ original ultrasound image (foetal image) given in (a) noisy image given in (b), median filter (median) in (c), speckle reducing anisotropic diffusion filtering (srad) in (d), geometric despeckle filter ( $g f 4 d)$ in (e), mean and variance local statistics despeckle filter (Ismv) in (f), wavelet thresholding in $(\mathrm{g})$, total variation despeckle filter (h), and proposed method in (i). 


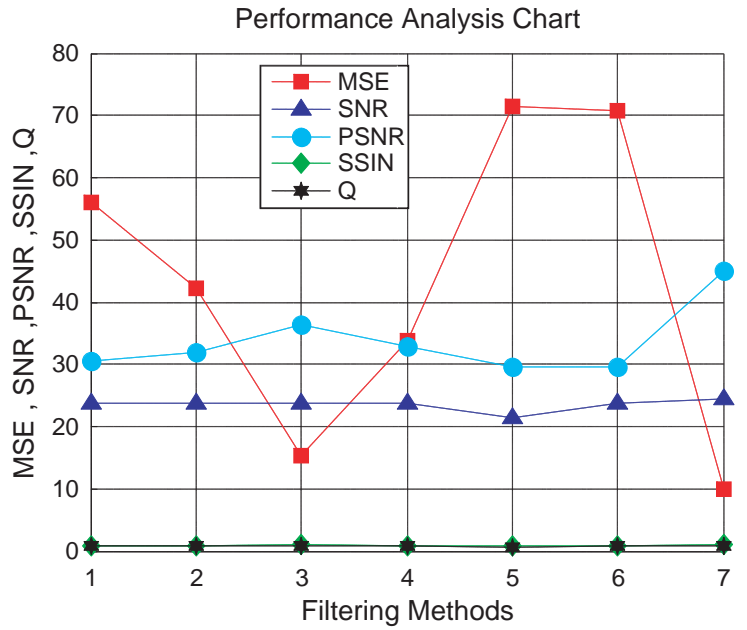

Fig. 12. Performance analysis graph, image quality evaluation metrics computed for the liver; at statistical measurements of MSE, SNR, PSNR and SSIN, $Q$; for different filter types to liver image, median filter (median) in (1), speckle reducing anisotropic diffusion filtering (srad) in (2), geometric despeckle filter $(g f 4 d)$ in (3), mean and variance local statistics despeckle filter (Ismv) in (4), wavelet thresholding in (5), total variation despeckle filter (6), and proposed method in (7).

despeckle $(g f 4 d)$, mean and variance local statistics despeckle $(l s m v)$, wavelet thresholding, and total variation despeckle filters. Simulation work was done on MATLAB R2008a (Mathworks, MA) running on an Intel Core-2 Due laptop with 2 GB of RAM.

Figure 2 shows example liver image subbands LL, HL, LH and $\mathrm{HH}$ obtained by wavelet decomposition using Daubechies "db8" family of wavelets. Figure 2(a) shows the low frequency subband LL, Figures 2(b)-(d) show the high frequency subbands of horizontal, vertical and diagonal directions, respectively. We can observe that most of the noise and texture are concentrated in the three high frequency subbands.

Figure 3 shows the modified wavelet coefficients (liver image) by our algorithm. In Figure 3, we can see that the LL subband is well denoised by TV method without losing texture information, and the textures in the three high frequency subbands are well extracted from the noise by wavelet thresholding and noise removed but edges smoothing and not preserved, after that we apply inverse wavelet transform to four subband and get the reconstructed image. In the last step we use TV method to get the final denoised images. Figures 4 and 5 show sample liver and trans-vaginal ultrasound images respectively, with speckle noise and results of various filters including the proposed method.

The results of the qualitative assessment are shown on Figures 4-8 for different types of images. As can be observed, the new method offers consistent reduction of the granularity of the speckle pattern without removing the texture or the important edges in the images. As an example, the parts of all images where there are blood vessels show cleaner lumen and edges with other parts showing finer speckle. Other methods suffer from blurring (such as (d) and (e) images in the above figures) or prominent vessel lumen texture (such as (b), (c), (f) and (g)).

The visual results of the quantitative assessment are shown in Figures 9-12 with their image quality evaluation metrics listed in Tables I-IV and plotted in Figures 13-16. It can be observed that the best visual results were obtained by the proposed method where noise was removed and details are preserved whereas the
Table I. Image quality evaluation metrics computed for the liver; at statistical measurements of MSE, SNR, PSNR and SSIN, Q; for different filter types to liver image.

\begin{tabular}{lccccc}
\hline & \multicolumn{5}{c}{ Image quality evaluation metrics } \\
\cline { 2 - 6 } Filter type & MSE $^{1}$ & SNR & PSNR & SSIN & Q \\
\hline Median & 56.0896 & 23.6660 & 30.6429 & 0.8682 & 0.7270 \\
Srad & 42.3332 & 23.6755 & 31.8640 & 0.8925 & 0.7249 \\
Gf4d & 15.2928 & 23.6667 & 36.2855 & 0.9912 & $\mathbf{0 . 8 6 8 1}$ \\
Lsmv & 33.7503 & 23.6668 & 32.8485 & 0.8856 & 0.7138 \\
Waveltc & 71.5293 & 21.4438 & 29.5858 & 0.7608 & 0.6590 \\
TV & 70.7416 & 23.6531 & 29.6335 & 0.8660 & 0.7021 \\
Proposed method & $\mathbf{1 0 . 0 2 3 5}$ & $\mathbf{2 4 . 5 1 6 3}$ & $\mathbf{4 5 . 0 6 9 4}$ & $\mathbf{0 . 9 9 1 4}$ & $\mathbf{0 . 8 5 3 5}$
\end{tabular}

Notes: Bold numbers indicate the best values. ${ }^{1} \mathrm{MSE}$, mean square error; SNR, signalto-noise ratio; PSNR, peak signal to-noise; SSIN, structural similarity index; $Q$, universal quality index.

Table II. Image quality evaluation metrics computed for trans-vaginal image type; at statistical measurements of MSE, SNR, PSNR and SSIN, $Q$; for different filter types to trans-vaginal image.

\begin{tabular}{lccccc}
\hline & \multicolumn{5}{c}{ Image quality evaluation metrics } \\
\cline { 2 - 6 } Filter type & MSE $^{1}$ & SNR & PSNR & SSIN & $\boldsymbol{Q}$ \\
\hline Median & 60.6296 & 23.8264 & 30.3040 & 0.9455 & 0.6793 \\
Srad & 60.1555 & 23.8105 & 30.3374 & 0.6953 & $\mathbf{0 . 6 9 3 1}$ \\
Gf4d & 59.1392 & $\mathbf{2 4 . 0 2 8 1}$ & 30.4119 & $\mathbf{0 . 9 9 6 4}$ & 0.6362 \\
Lsmv & 58.9763 & 23.8275 & 30.4238 & 0.7947 & 0.6665 \\
Waveltc & 40.8768 & 23.8135 & 32.0167 & 0.8109 & 0.6476 \\
TV & 59.0316 & 23.8211 & 30.4206 & 0.4565 & 0.6465 \\
Proposed method & $\mathbf{2 5 . 0 5 4 0}$ & $\mathbf{2 3 . 8 3 3 4}$ & $\mathbf{3 4 . 1 4 1 1}$ & $\mathbf{0 . 9 4 7 8}$ & $\mathbf{0 . 6 8 9 5}$ \\
\hline
\end{tabular}

Notes: Bold numbers indicate the best values. ${ }^{1} \mathrm{MSE}$, mean square error; SNR, signalto-noise ratio; PSNR, peak signal to-noise; SSIN, structural similarity index; $Q$, universal quality index.

Table III. Image quality evaluation metrics computed for pelvic image type; at statistical measurements of MSE, SNR, PSNR and SSIN, Q; for different filter types to liver image.

\begin{tabular}{lccccc}
\hline & \multicolumn{5}{c}{ Image quality evaluation metrics } \\
\cline { 2 - 6 } Filter type & MSE $^{1}$ & SNR & PSNR & SSIN & Q \\
\hline Median & 94.0337 & 21.6879 & 28.3975 & 0.8144 & 0.9732 \\
Srad & 93.8167 & 21.7009 & 28.4086 & 0.8754 & 0.9817 \\
Gf4d & 94.3365 & 21.6723 & 28.3847 & 0.7517 & 0.9766 \\
Lsmv & 94.2491 & 21.6876 & 28.3884 & 0.7075 & 0.9879 \\
Waveltc & 94.0764 & 21.7074 & 28.3950 & 0.8204 & 0.9532 \\
TV & 94.3439 & 21.6307 & 28.3975 & 0.6988 & 0.9412 \\
Proposed method & $\mathbf{8 1 . 9 7 4 9}$ & $\mathbf{2 2 . 7 3 3 9}$ & 28.9946 & $\mathbf{0 . 9 0 6 1}$ & $\mathbf{0 . 9 8 9 9}$ \\
\hline
\end{tabular}

Notes: Bold numbers indicate the best values. ${ }^{1} \mathrm{MSE}$, mean square error; SNR, signalto-noise ratio; PSNR, peak signal to-noise; SSIN, structural similarity index; $Q$, universal quality index.

Table IV. Image quality evaluation metrics computed for fetus image type; at statistical measurements of MSE, SNR, PSNR and SSIN, Q; for different filter types to liver image.

\begin{tabular}{lccccc}
\hline & \multicolumn{5}{c}{ Image quality evaluation metrics } \\
\cline { 2 - 6 } Filter type & MSE & SNR & PSNR & SSIN & Q \\
\hline Median & 41.0023 & 23.7426 & 32.0020 & 0.8902 & 0.9947 \\
Srad & 49.9382 & 23.7454 & 31.1460 & 0.8811 & 0.9938 \\
Gf4d & 64.3220 & 23.7340 & 30.0480 & 0.8741 & 0.9940 \\
Lsmv & 77.3133 & 23.7402 & 29.2480 & 0.8532 & 0.9945 \\
Waveltc & 34.8797 & 23.7466 & 32.7060 & 0.8947 & 0.9947 \\
TV & 25.8764 & 23.7396 & 34.0020 & 0.8991 & 0.9956 \\
Proposed method & $\mathbf{2 4 . 1 6 8 9}$ & $\mathbf{2 4 . 2 0 3 1}$ & $\mathbf{3 4 . 2 9 8 0}$ & $\mathbf{0 . 9 2 0 1}$ & $\mathbf{0 . 9 9 8 5}$
\end{tabular}

Notes: Bold numbers indicate the best values. ${ }^{1} \mathrm{MSE}$, mean square error; SNR, signalto-noise ratio; PSNR, peak signal to-noise; SSIN, structural similarity index; $Q$, universal to-noise ratio; 


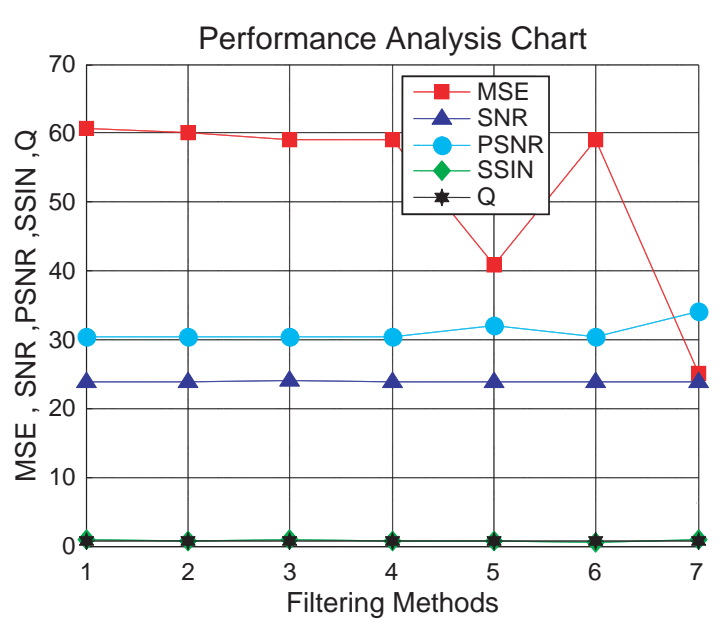

Fig. 13. Performance analysis graph, image quality evaluation metrics computed for the trans-vaginal; at statistical measurements of MSE, SNR, PSNR and SSIN, $Q$; for different filter types to liver image, median filter (median) in (1), speckle reducing anisotropic diffusion filtering (srad) in (2), geometric despeckle filter $(g f 4 d)$ in $(3)$, mean and variance local statistics despeckle filter (Ismv) in (4), wavelet thresholding in (5), total variation despeckle filter (6), and proposed method in (7).

results of wavelet thresholding shows blurring of edges, and those by TV method retained most of the speckle noise. From the quality evaluation metric in the tables and plots, excellent performance was shown for the proposed method with lower MSE and higher SNR and PSNR and best values for the structural similarity index, SSIN and the universal quality index, $Q$. Filters such as median, $l s m v$, waveltc, $T V$, showed poorer visual results and a blurring effect; but the filter $g f 4 d$; with best value for $Q$, shows better visual results in liver image Table I, and filters $\mathrm{srad}$; with best value for $Q, g f 4 d$; with high SNR, and best value for SSIN, shows better visual results in Trans-vaginal image in Table II. It has been found that the proposed method is generally better in quantitative terms as well as visual quality of the image.

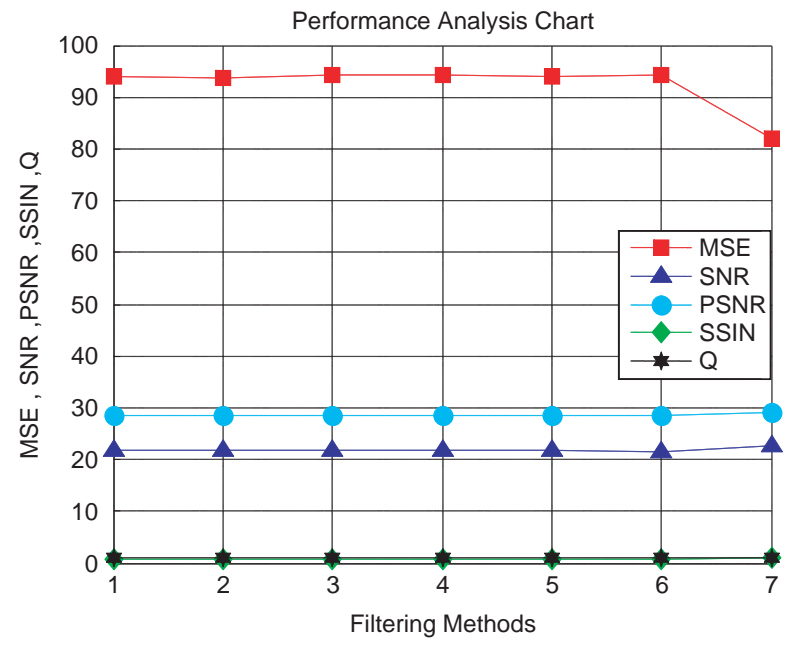

Fig. 14. Performance analysis graph, image quality evaluation metrics computed for the pelvic; at statistical measurements of MSE, SNR, PSNR and SSIN, $Q$; for different filter types to liver image, median filter (median) in (1), speckle reducing anisotropic diffusion filtering (srad) in (2), geometric despeckle filter $(g f 4 d)$ in (3), mean and variance local statistics despeckle filter (Ismv) in (4), wavelet thresholding in (5), total variation despeckle filter (6), and proposed method in (7).

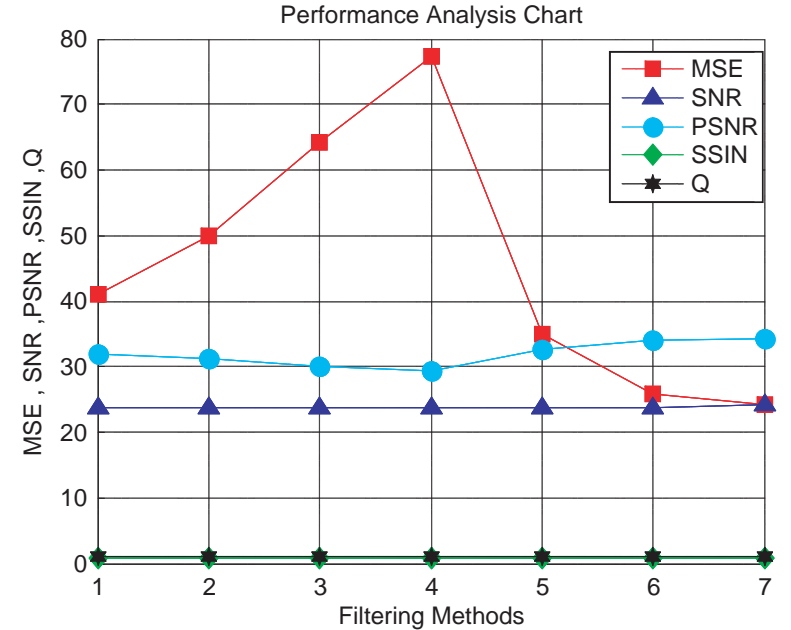

Fig. 15. Performance analysis graph, image quality evaluation metrics computed for a fetus image; at statistical measurements of MSE, SNR, PSNR and SSIN, $Q$; for different filter types to liver image, median filter (median) in (1), speckle reducing anisotropic diffusion filtering (srad) in (2), geometric despeckle filter $(g f 4 d)$ in (3), mean and variance local statistics despeckle filter (Ismv) in (4), wavelet thresholding in (5), total variation despeckle filter (6), and proposed method in (7).

\section{DISCUSSION}

The issue of choosing the wavelet family to use in different problems is not resolved in general. In our problem, our experiments did not show major differences between the different wavelet families that we tried and we do not have conclusive evidence that one of them is better. So, the "db8" wavelet used here can be considered as a specification to "one" working version of the method so that there are no missing steps for complete implementation.

The experimental evaluation of the new method compared the method to six different classes of filtering methods. Even though hybrids of these methods exist, we preferred to compare to the fundamental methods since our implementation of several such hybrids did not yield good results (likely due to need for parameter tuning). In general, the new method was shown to preserve edges and fine details like textures while removing the noise as indicated by the experimental results. Initial findings show promising results; however, further clinical work is required to evaluate the utility of the proposed method to assess its impact in clinical practice.

\section{CONCLUSIONS}

A new speckle reduction method combining the TV method and wavelet soft thresholding is developed. The new method combines the denoising property of wavelet thresholding algorithm with the total variation model. The total variation based method is used to denoise the low frequency wavelet subband without losing texture, and to reduce the oscillations that may be generated around the edges when use wavelet thresholding. On the other hand, the wavelet thresholding method is used in the high frequency subbands where it is very efficient in representing the smooth parts of an image and is the ability to treat different frequency components of an image separately. The developed method was evaluated using both qualitative and quantitative studies and the results indicate a potential for the utility of the new method in clinical settings. 
Acknowledgment: The Matlab code used to verify the previous methods was a modified version of the code found in Refs. [42-43]. We also would like to thank International Biomedical Engineering Technologies (Giza, Egypt) for making their database of ultrasound images available to the authors for testing their methods and for also helping evaluate the results.

\section{References and Notes}

1. A. Webb, Introduction to Biomedical Imaging, John Wiley \& Sons, Hoboken (2003)

2. A. K. Jain, Fundamental of Digital Image Processing, Prentice-Hall, Englewood (1989).

3. C. B. Burckhardt, Speckle in ultrasound B-mode scans. IEEE Trans. Sonics Ultrason. SU-25, 1 (1978).

4. R. F. Wagner, S. W. Smith, J. M. Sandrik, and H. Lopez, Statistics of speckle in ultrasound B-scans. IEEE Trans. Sonics Ultrason. 30, 156 (1983).

5. J. W. Goodman, Some fundamental properties of speckle. J. Opt. Soc. Amer. 66, 1145 (1976).

6. Y. Yongjian and S. T. Acton, Speckle reducing anisotropic diffusion. IEEE Trans. Image Processing 11, 1260 (2002).

7. R. W. Prager, A. H. Gee, G. M. Treece, and L. Berman, Speckle detection in ultrasound images using first order statistics, University of Cambridge, Department of Engineering, Internal Report (2002), Vol. 415, p. 1.

8. S. K. Narayanan and R. S. D. Wahidabanu, A view on despeckling in ultrasound imaging. International Journal of Signal Processing, Image Processing and Pattern Recognition 2, 85 (2009).

9. T. Huang, G. Yang, and G. Tang, A fast two-dimensional median filtering algorithm. IEEE Trans. Acoust. Speech Signal Processing 27, 13 (1979).

10. Y. Yongjian and S. T. Acton, Speckle reducing anisotropic diffusion. IEEE Trans. Image Processing 11, 1260 (2002).

11. L. Busse, T. R. Crimmins, and J. R. Fienup, A model based approach to improve the performance of the geometric filtering speckle reduction algorithm. Proc. 1995 IEEE Int. Ultrason. Symp. 1353 (1995).

12. A. Achim, A. Bezerianos, and P. Tsakalides, Novel Bayesian multiscale method for speckle removal in medical ultrasound images. IEEE Trans. Med. Imag. 20, 772 (2001).

13. J. F. Aujol and A. Chambolle, Dual norms and image decomposition models. International Journal on Computer Vision 63, 85 (2005).

14. L. Rudin, S. Osher, and E. Fatemi, Nonlinear total variation based noise removal algorithms. Physica D 60, 259 (1992).

15. A. Chambolle, An algorithm for total variation minimization and applications. Journal of Mathematical Imaging and Vision 20, 89 (2004).

16. S. Osher, A. Sole, and L. Vese, Image decomposition and restoration using total variation minimization and the $\mathrm{H}-1$ norm. SIAM Journal on Multiscale Modeling and Simulation 1, 349 (2003).

17. G. Gilboa, N. Sochen, and Y. Y. Zeevi, Texture preserving variational denoising using an adaptive fidelity term. IEEE Transactions on Image Processing 15, 2281 (2006).

18. $Y$. Meyer, Oscillating patterns in image processing and nonlinear evolution equations, The Fifteenth Dean Jacque Lines $B$, Lewis Memorial Lectures, American Mathematical Society Press, Rhode Island (2002).
19. D. L. Donoho and I. M. Johnstone, Denoising by soft thresholding. IEEE Transactions on Information Theory 41, 613 (1995)

20. D. L. Donoho and I. M. Johnstone, Ideal spatial adaptation by wavelet shrinkage. Biomerika 81, 425 (1994)

21. D. L. Donoho and I. M. Johnstone, Adapting to unknown smoothness via wavelet shrinkage. J. Amer. Statist. Assoc. 90, 1200 (1995).

22. S. Chang, B. Yu, and M. Vetterli, Adaptive wavelet thresholding for image denoising and compression. IEEE Transactions on Image Processing 9, 1532 (2000).

23. A. Chambolle, R. Devore, N. Lee, and B. J. Lucier, Nonlinear wavelet image processing: Variational problems, compression, and noise removal through wavelet shrinkage. IEEE Transactions on Image Processing 7, 319 (1998).

24. I. Daubechies, The wavelet transform, time-frequency localization and signal analysis. IEEE Transactions on Information Theory 36, 961 (1990)

25. Z. Tao and F. Qibin, Texture preserving denoising method combining total variation and wavelet shrinkage. Journal of Natural Sciences 15, 031 (2010).

26. S. Roy, N. Sinha, and A. K. Sen, A new hybrid image denoising method. International Journal of Information Technology and Knowledge Management 2, 491 (2010).

27. S. Durand and J. Froment, Reconstruction of wavelet coefficients using total variation minimization. SIAM Journal on Scientific Computing 24, 1754 (2003).

28. R. R. Coifman and A. Sowa, Combing the calculus of variations and wavelets for image enhancement. Appl. Compt. Harmon. Ana. 9, 1 (2000)

29. T. F. Chan and H. M. Zhou, Total variation improved wavelet thresholding in image compression, Proc. Seventh International Conference on Image Processing, IEEE Press (2000), pp. 391-394.

30. G. Steidl, J. Weickert, T. Brox, T. Mrazek, and M. Welk, On the equivalence of soft wavelet shrinkage, total variation diffusion, total variation regularization, and sides. SIAM Journal on Numerical Analysis 42, 686 (2004).

31. I. Daubechies and G. Teschke, Variational image restoration by means of wavelets: Simultaneous decomposition, deblurring, and denoising. Applied and Computational Harmonic Analysis 19, 1 (2005)

32. S. Sudha, G. R. Suresh, and R. Sukanesh, Speckle noise reduction in ultrasound images by wavelet thresholding based on weighted variance. International Journal of Computer Theory and Engineering 1, 1793 (2009).

33. T. J. Chen, K. S. Chuang, J. Wu, S. C. Chen, I. M. Hwang, and M. L. Jan, A novel image quality index using Moran I statistics. Phys. Med. Biol. 48, 131 (2003).

34. D. Sakrison, On the role of observer and a distortion measure in image transmission. IEEE Trans. Comm. 25, 1251 (1977).

35. Z. Wang, A. Bovik, H. Sheikh, and E. Simoncelli, Image quality assessment: From error measurement to structural similarity. IEEE Trans. Image Processing 13, 600 (2004).

36. Z. Wang and A. Bovik, A universal quality index. IEEE Signal Processing Lett. 9, 81 (2002).

37. http://www.medison.ru/uzi/eho240.htm

38. http://www.ibetech.com.

39. http://www.medison.ru/uzi/eho240.htm

40. http://www.ultrasoundcare.com.au.

41. http://www.rusiadiagnosticcentre.com.

42. C. P. Loizou and C. S. Pattichis, Despeckle Filtering Algorithms and Software for Ultrasound Imaging, Morgan and Claypool Publishing (2008)

43. http://www.medinfo.cs.ucy.ac.cy/index.php/downloads/toolboxes. 